\title{
Photocatalytic polymerization induced by a transparent anatase titania aqueous sol and fabrication of polymer composites
}

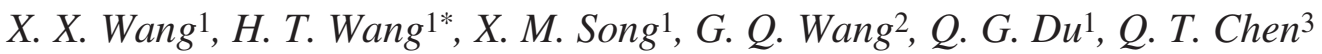 \\ ${ }^{1}$ Key Laboratory of Molecular Engineering of Polymers of Ministry of Education, Department of Macromolecular \\ Science, Fudan University, Shanghai 200433, People's Republic of China \\ 2Innovative Systems and Materials Laboratory, Shanghai Research Institute, Hitachi (China) Research \& Development \\ Corporation, Shanghai 200020, People's Republic of China \\ ${ }^{3}$ Shanghai Koito Automotive Lamp Co., Ltd, Shanghai 201821, People's Republic of China
}

Received 20 November 2009; accepted in revised form 23 April 2010

\begin{abstract}
The surface modification of the anatase titania nanoparticles prepared via a controlled nonhydrolytic sol-gel process is achieved by the formation of the bidentate coordination between titania and methacrylic acid (MAA) molecules. The in situ photocatalytic polymerization of methyl methacrylate (MMA) monomer is initiated by surface modified anatase titania nanoparticles under Xe lamp irradiation. A variety of techniques including differential scanning calorimetry (DSC), thermo-gravimetric analysis (TGA) and scanning electron microscopy (SEM) are employed to characterize the resulting materials. The glass transition temperatures and the thermal stabilities of polymethyl methacrylate (PMMA) composite materials prepared via photocatalytic polymerization are enhanced compared with pure polymer. The partial aggregation of titania nanoparticles in PMMA composite films is derived from the surface polymerization of MMA, which makes the inorganic particles hydrophobic and drives them to the water/oil interfaces.
\end{abstract}

Keywords: polymer composites, thermal properties, titania, morphologies

\section{Introduction}

At present, titania has been considered as one of the most studied semiconductors and widely used in gas sensors [1], as a pigment [2], in solar-energy conversion $[3,4]$ and as a promising photocatalyst for water and air purification [5] because of its high oxidative power, chemical stability, low cost and nontoxicity [6]. Titania is also used as a photocatalyst to initiate polymerization of vinyl monomers without adding any photosensitizers, which has acquired important progresses in this aspect [7-9]. Ni et al. [7] studied the kinetics of the photopolymerization of methyl methacrylate initiated by titania nanopowders in aqueous suspensions. Damm and coworkers $[8,9]$ reported the influence of the properties of titania on the photocatalytic acrylate polymerization and also investigated the photopolymerization of an ethoxylated trisacrylate initiated by iron-doped titania particles. Most of the research articles concerned the photocatalytic polymerization induced by crystalline titania powders. A transparent anatase titania aqueous sol, however, as the photocatalyst to initiate polymerization of vinyl monomers has hardly ever been studied. The titania nanoparticles in the sol normally have smaller particle size (less than several tens nanometers) and less aggregation because of hydrophilic surfaces and electrostatic repulsion of nanoparticles. The in situ photocatalytic polymerization induced by titania aqueous sol may decrease the 
aggregation of inorganic nanoparticles in polymer matrices. With the increasing interests in the preparation of polymer/inorganic nanocomposites, including polymer/titania composite materials $[10,11]$, the homogeneous dispersion of the inorganic nanoparticles in the polymer matrices avoiding macroscopic phase separation is very important at any time. Therefore, the surface functionalization of titania nanoparticles has attracted increasing attention. There have been several methods for the modification of titania particles. One is using a silane coupling agent to functionalize nanosized titania [12-14]. Second is encapsulating titania particles within organic polymers by various polymerization methods [15-17]. The third method is surface modification of the titania nanoparticles with bifunctional molecules $[18,19]$.

In this work, we report a practical and convenient method to prepare a stable and transparent anatase titania aqueous sol. This preparation method was based on a nonhydrolytic sol-gel process using titanium tetrachloride as precursor in ethanol solution. The solution was then dried at $80^{\circ} \mathrm{C}$ to produce anatase titania nanoparticles and they were dispersed in water to obtain the titania sols. After functionalization of the anatase titania surface with methacrylic acid coupling agent, the PMMA/titania composite materials were synthesized through an in situ photocatalytic polymerization of methyl methacrylate under Xe lamp irradiation. This preparation method is environmentally friendly approach because no organic solvent is used in the polymerization process. The influence of surface modification of anatase titania nanoparticles on the morphology and thermal properties of PMMA composite materials was investigated. A tentative mechanism for the partial aggregation of titania nanoparticles during photocatalytic polymerization process was also presented.

\section{Experimental}

\subsection{Materials}

Titanium (IV) chloride $\left(\mathrm{TiCl}_{4}\right.$, chemical reagent grade), ethanol (EtOH, analytical reagent grade), methanol $(\mathrm{MeOH}$, analytical reagent grade), methyl methacrylate (MMA, analytical reagent grade), azobisisobutyronitrile (AIBN, chemical reagent grade), methacrylic acid (MAA, analytical reagent grade) and tetrahydrofuran (THF, analyti- cal reagent grade) were purchased from Shanghai Chemical Reagent Company, Shanghai, China. MMA was purified by distillation and AIBN was recrystallized before use. $\mathrm{TiCl}_{4}$, ethanol, MAA, methanol and THF were used without further purification. Deionized water was used in the experiments.

\subsection{Synthesis of anatase titania sol}

The anatase titania sol was prepared by a convenient nonhydrolytic sol-gel method. Briefly, $\mathrm{TiCl}_{4}$ was slowly added to anhydrous ethanol under vigorous stirring. The mixture was stirred magnetically at room temperature for $12 \mathrm{~h}$ and then dried at $80^{\circ} \mathrm{C}$ for $20 \mathrm{~h}$ to produce off-white powders. They were grinded by a ball mill (QM-3SP04, Nanjing University Instrument Plant, Nanjing, China) at room temperature for $24 \mathrm{~h}$ and the nanosized titania particles were obtained. The $5 \mathrm{wt} \%$ anatase titania sol was produced after dispersion of $1 \mathrm{~g}$ titania nanoparticles in $19 \mathrm{~g}$ deionized water.

\subsection{Surface modification of anatase titania nanoparticles}

The surface modification of anatase titania nanoparticles was performed by mixing $20 \mathrm{~g} 5 \mathrm{wt} \%$ anatase titania sol with different contents $(0.11$ or $0.25 \mathrm{~g})$ of MAA coupling agent. The solution was then stirred magnetically at room temperature for $12 \mathrm{~h}$ in dark. The content of MAA in titania/MAA system was 10 and $20 \mathrm{wt} \%$, respectively.

\subsection{Preparation of PMMA/titania composite materials}

The PMMA/titania composite materials were prepared by in situ photocatalytic polymerization of MMA in the presence of the surface modified anatase titania sol. The mixtures of $9 \mathrm{~g}$ MMA monomer and $20 \mathrm{~g}$ above mentioned surface modified anatase titania sol were deoxygenated by bubbling with nitrogen for $20 \mathrm{~min}$. Then the glass flasks were sealed and the polymerization was carried out by exposing the flask to a XQ 500W Xe lamp (the distance of the glass flasks to the Xe lamp was $20 \mathrm{~cm}$ ) with continuous stirring for $48 \mathrm{~h}$ at room temperature. The products were filtered and dissolved in a good solvent of PMMA (THF) fol- 
lowed by being precipitated in water. And then they were filtered and dried at $50^{\circ} \mathrm{C}$ for $48 \mathrm{~h}$ to obtain the PMMA/titania composite materials. For comparison, the polymerization of MMA using unmodified titania sol was also performed under the same experimental conditions.

\subsection{Preparation of PMMA homopolymer}

For comparison, PMMA homopolymer was also prepared via a conventional free radical polymerization described as follows. The monomer MMA and the initiator AIBN (0.2 wt \% in the monomers) were added to a reaction flask and then polymerized at $70^{\circ} \mathrm{C}$ under nitrogen atmosphere for $12 \mathrm{~h}$. The resulting polymer was purified by precipitating in methanol and then dried at room temperature under vacuum. The molecular weight $\left(M_{w}\right)$ of PMMA was $\sim 120000 \mathrm{~g} / \mathrm{mol}$ and its polydispersity (D) was 1.8 .

\subsection{Preparation of PMMA/titania composite films}

To prepare PMMA/titania composite films, $2 \mathrm{~g}$ composite materials were dissolved in $15 \mathrm{~g}$ THF by vigorous stirring and the mixture was cast on a polyethylene terephthalate (PET) substrate and dried at $50^{\circ} \mathrm{C}$ in the oven for $10 \mathrm{~h}$. For comparison, the PMMA/titania material via subsequent addition of titania was also prepared. 1.8 g PMMA homopolymer was dissolved in $15 \mathrm{~g}$ THF by vigorous stirring followed by the addition of $0.2 \mathrm{~g}$ the assynthesized titania nanoparticles with continuous stirring for $2 \mathrm{~h}$. Then the mixture was also cast on a PET substrate and dried at $50^{\circ} \mathrm{C}$ in the oven for $10 \mathrm{~h}$. The thickness of these composite films was in the range of $50-60 \mu \mathrm{m}$.

\subsection{Measurements}

FT-IR spectra were measured on a Nicolet NEXUS-470 spectrometer. The pure and modified titania nanoparticles were dried and washed with ethanol carefully. Then the powders were dried in a vacuum oven and pressed into $\mathrm{KBr}$ pellets for FTIR measurements. The absorption spectra of anatase titania sol was recorded using a Perkin-Elmer Lambda 35 UV/Vis spectrometer. The thermal stability of pure PMMA and the PMMA/titania com- posite materials was investigated by thermo-gravimetric analysis (TGA) using a Perkin-Elmer Pyris 1 thermogravimetric analyzer. The measurements were conducted at a heating rate of $20^{\circ} \mathrm{C} / \mathrm{min}$ in air atmosphere. Differential scanning calorimetry (DSC) tests of pure PMMA and the composite materials were performed at a heating rate of $10^{\circ} \mathrm{C} / \mathrm{min}$ in the nitrogen atmosphere using a Perkin-Elmer DSC-7 apparatus. Transmission electron microscopy (TEM) investigations were performed on a Hitachi H-600 microscope. The samples for TEM observation were prepared by adding 1-2 drops of the anatase titania sol on lacey carbon copper grids. The X-ray powder diffraction (XRD) patterns were measured on an X'Pert PRO (PANalytical) diffractometer at room temperature with $\mathrm{CuK}_{\alpha}$ radiation. XRD diagrams were obtained for the range of $2 \theta=20-80^{\circ}$. The morphologies of the fractured surfaces of the PMMA/titania composite films were observed with a JSM-5600LV and SUPERSCAN SS-550 scanning electron microscope (SEM). The particle size and particle size distribution were determined by measurement of about 300 particles. Gel permeation chromatographic (GPC, Agilent 1100, USA) analysis was carried out at $30^{\circ} \mathrm{C}$ at a flow rate of $1 \mathrm{ml} / \mathrm{min}$ using THF as the solvent. The instrument was calibrated using polystyrene as standard.

\section{Results and discussion}

\subsection{Properties of the anatase titania sol}

Figure 1 shows the UV/Vis spectrum of the transparent anatase titania sol. It can be found that the sol has a strong absorption in UV region, while it is

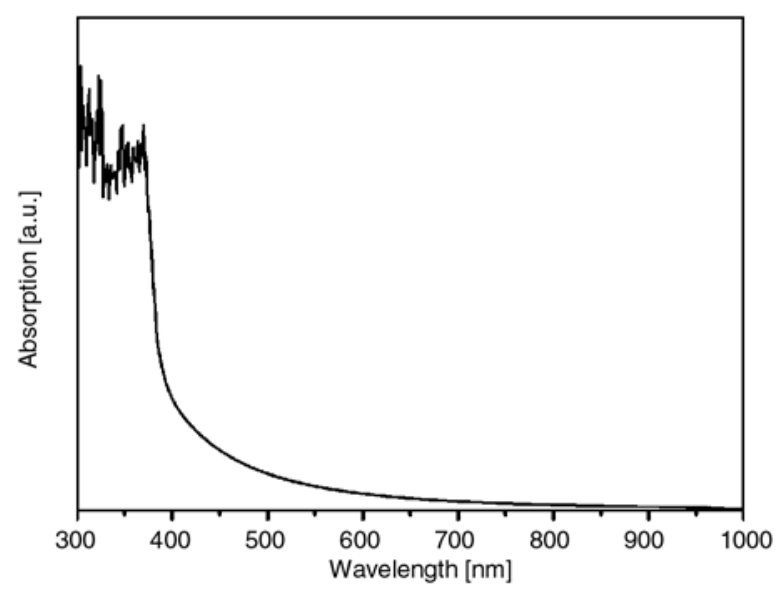

Figure 1. UV/Vis spectrum of the prepared anatase titania sol 


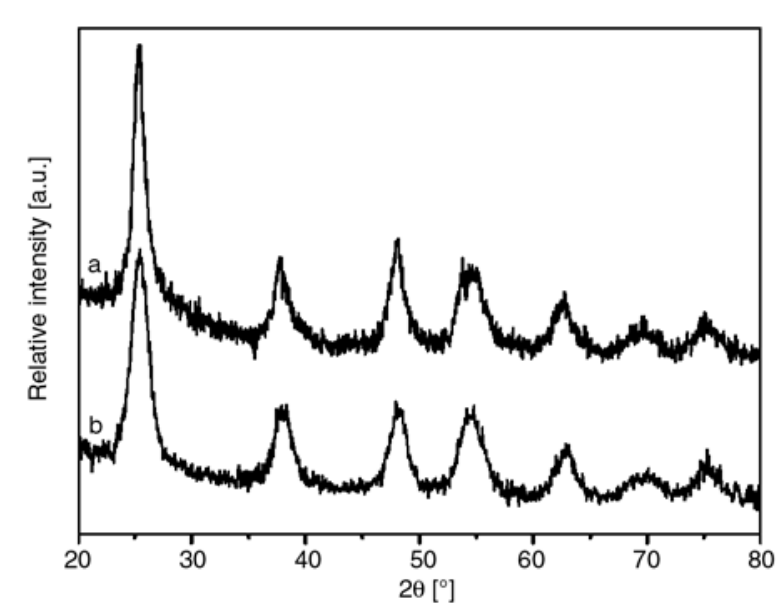

Figure 2. XRD patterns of (a) original titania nanoparticles and (b) the dried titania sol

almost transparent in visible region. This is in accordance with its appearance detected by eye. No precipitation is found after 3 months, which indicates that the anatase titania sol is very stable at room temperature.

Figure 2 presents the XRD patterns of the original titania nanoparticles obtained by the controlled nonhydrolytic sol-gel process and the dried titania sol. It is seen that there is almost no difference in the XRD patterns between these two kinds of nanoparticles. They both show well-defined diffraction peaks characteristic of anatase phase [20] and the crystal size determined by the Scherrer equation is about $9 \mathrm{~nm}$. These facts suggest that

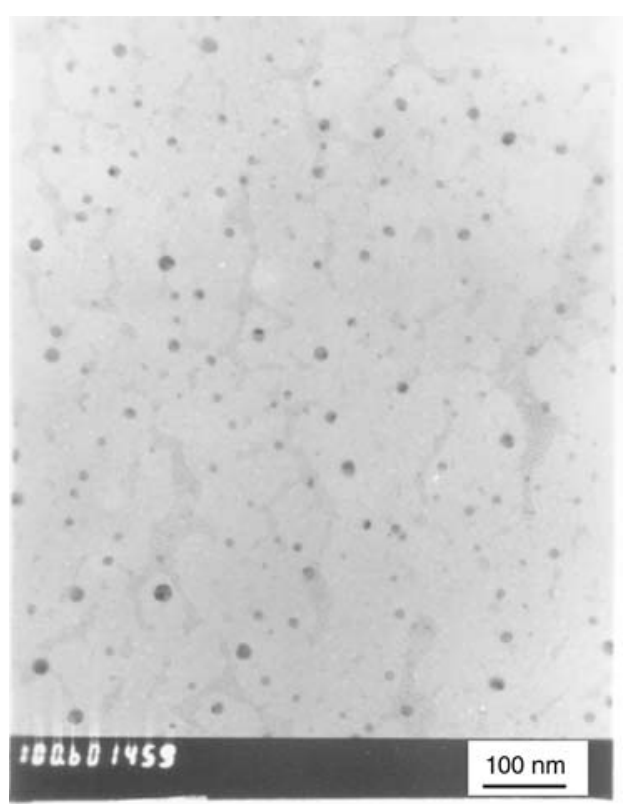

Figure 3. TEM image of the prepared anatase sol crystallinity of the anatase titania nanoparticles is not affected after dispersion in water.

Transmission electron microscopy (TEM) was used to examine the size of anatase titania sol as shown in Figure 3. The average size of the titania nanoparticles in sol is around $20 \mathrm{~nm}$, which is bigger than the crystal size determined by XRD. This is due to the incomplete crystallinity of these titania nanoparticles. The anatase titania nanoparticles may have applications in the photocatalytic polymerization and the preparation of polymer/titania nanocomposites.

\subsection{FT-IR spectra of the modified anatase titania nanoparticles}

The FT-IR spectra of anatase titania sol modified with different content of MAA after careful rinse are shown in Figure 4. The original titania particles (Figure 4a) have no absorption peaks from 1400 to $1800 \mathrm{~cm}^{-1}$ except a strong bands at $1630 \mathrm{~cm}^{-1}$ which is the characteristic of bending vibration of water molecules [21]. For the samples after functionalized by MAA coupling agent (Figure $4 \mathrm{~b}$ and $4 c$ ), the peak at $1721 \mathrm{~cm}^{-1}$ is attributed to typical $\mathrm{C}=\mathrm{O}$ vibration [22] and the bands at 1540, 1440, and $1410 \mathrm{~cm}^{-1}$ are due to the bidentate coordination between titanium centers and the carboxylic groups of MAA [19]. The FT-IR spectra confirm that the coupling agent is successfully bonded with the titania nanoparticles, which is used to improve the compatibility of PMMA and inorganic moiety.

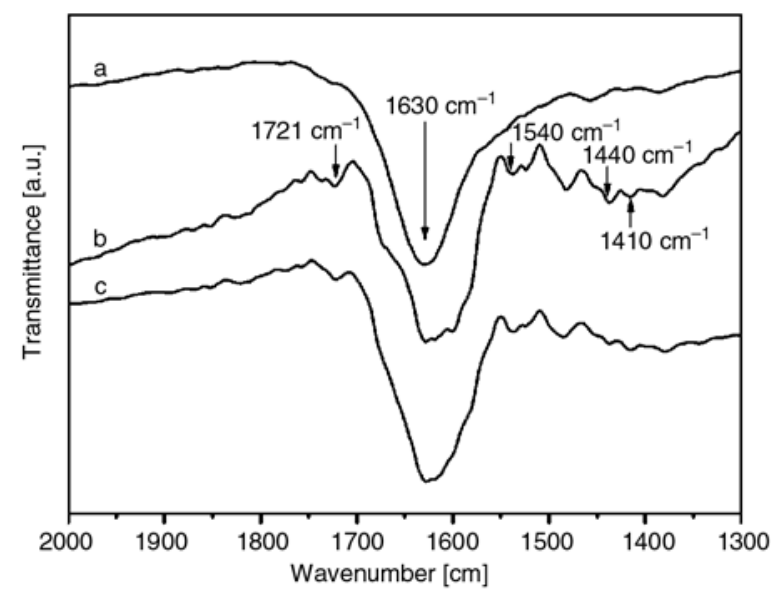

Figure 4. FTIR spectra of anatase titania sol modified with different content of MAA: (a) 0, (b) 10 and (c) $20 \mathrm{wt} \%$ 


\subsection{Glass transition temperatures of PMMA/titania composites}

Figure 5 shows the DSC curves of the pure PMMA and the PMMA/titania composite materials at a heating rate of $10^{\circ} \mathrm{C} / \mathrm{min}$ under nitrogen flow. All PMMA/titania composites obtained by photocatalytic polymerization exhibit an increased glass transition temperature $\left(T_{g}\right)$ compared with pure PMMA (Figure $5 \mathrm{a}, T_{g} \sim 111^{\circ} \mathrm{C}$ ), which indicates the restrained mobility of the polymer chains in the composites. This is attributed to the intercalated polymer chains within titania particles that prevents their segmental motion. Moreover, the higher $T_{g}$ value of PMMA may also depend on the predominant syndiotacticity of the polymer prepared by photocatalytic polymerization [7]. The PMMA/titania composite obtained via photocatalytic polymerization by original anatase titania sol exhibits a glass transition temperature of $123^{\circ} \mathrm{C}$ (Figure 5b), while the PMMA-functionalized titania composites show elevated $T_{g}$ values ranging from 127 to $129^{\circ} \mathrm{C}$ (Figure $5 \mathrm{c}$ and $5 \mathrm{~d}$ ). Therefore, the interfacial strength becomes stronger by the addition of MAA coupling agent. These DSC results indicate that the PMMA produced by photocatalytic polymerization has surely enhanced glass transition temperature. And moreover, the $T_{g}$ values of PMMA composite materials is further increased by using MAA as coupling agent due to the formation of much stronger interactions between PMMA molecules and titania nanoparticles.

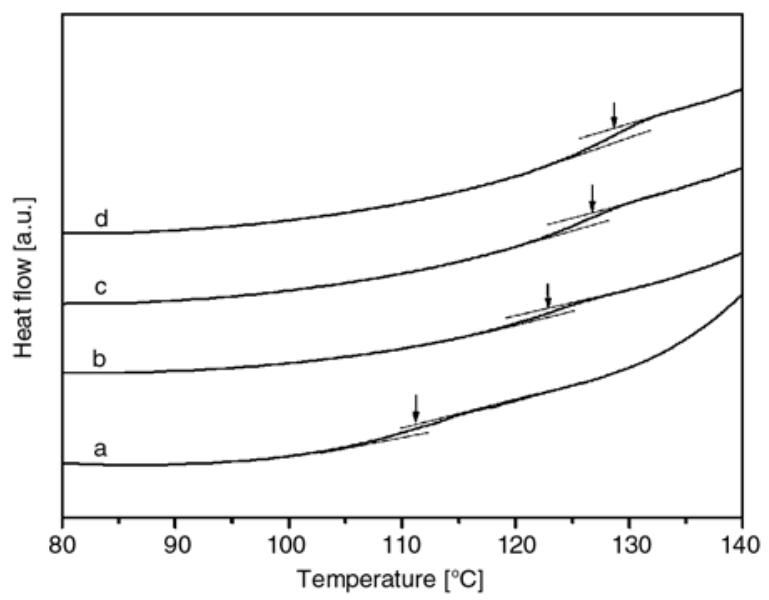

Figure 5. DSC curves of (a) pure PMMA and the composite materials with different MAA contents: (b) 0 , (c) 10 and (d) $20 \mathrm{wt} \%$

\subsection{Thermal stabilities of PMMA/titania composites}

The TGA curves of a pure PMMA and PMMA/titania composite materials with $10 \mathrm{wt} \%$ inorganic nanoparticles modified using different content of MAA as coupling agent are shown in Figure 6. In general, for the thermal decomposition of pure PMMA (Figure 6a), at least two degradation steps are clearly found and it starts decomposition at about $150^{\circ} \mathrm{C}$. Previous works has proved that there are two steps in the thermo-oxidative degradation of PMMA. The first is due to the degradation of partially oxidized groups. The second is an attack of oxygen on the free radicals generated by random scission within the polymer chain $[23,24]$. While there appears to be one step of weight loss for the composite materials starting at about $250^{\circ} \mathrm{C}$ and ending at about $400^{\circ} \mathrm{C}$, which corresponds to the structural decomposition of the polymers. According to our previous work, the thermo-oxidative degradation mechanism of the PMMA/titania composite materials is such that titania may trap the free radical in the process of thermo-oxidative degradation of PMMA [25]. So evidently, the thermal decomposition of these composite materials shifts toward much higher temperature range than that of pure PMMA, which confirms the enhancement of thermal stability of PMMA by the introduction of titania nanoparticles. Furthermore, we clearly found that the $T_{0.5}$ ( $T_{0.5}$ stands for the temperature at which $50 \%$ weight loss took place) of the

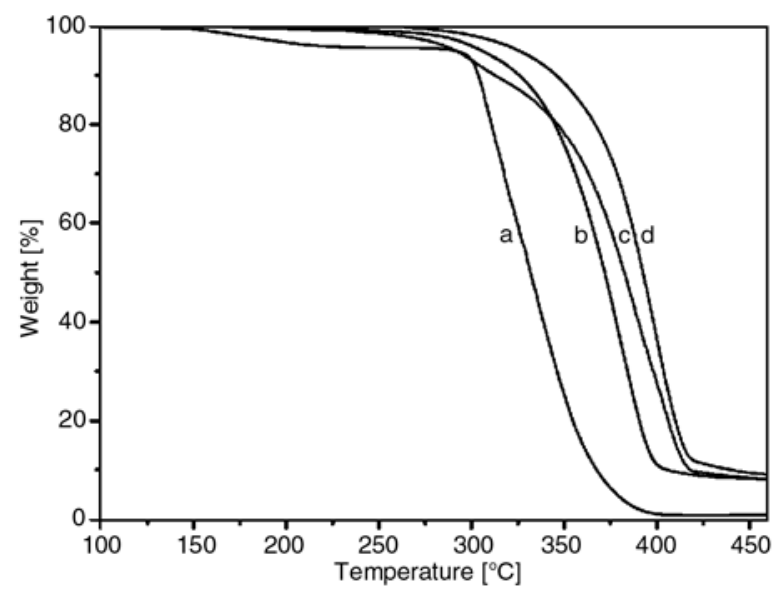

Figure 6. TGA curves of (a) pure PMMA and PMMA/titania composite materials with different content of MAA as coupling agent: (b) 0 , (c) 10 and (d) $20 \mathrm{wt} \%$ 
PMMA/titania composite materials with 0 (Figure 6b), 10 (Figure 6c) and 20wt \% (Figure 6d) MAA as coupling agent are 372,382 and $392^{\circ} \mathrm{C}$ respectively. So, when compared with PMMA/nonfunctional titania composites, the thermal properties of the as-synthesized PMMA/titania composite materials with MAA as coupling agent are enhanced. This implies that the use of MAA as coupling agent

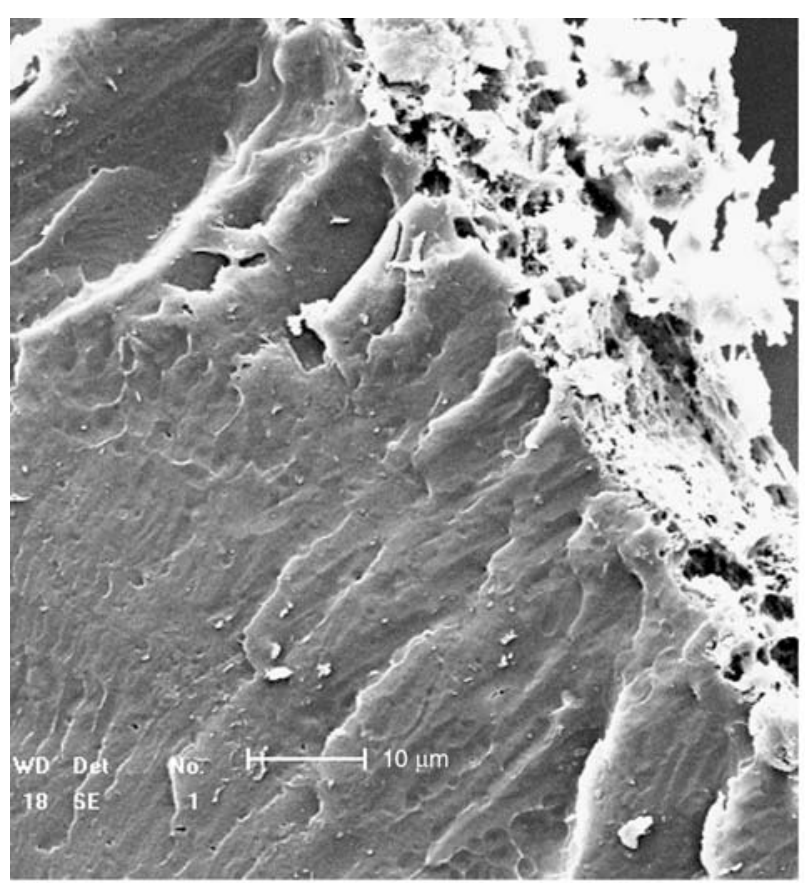

a)

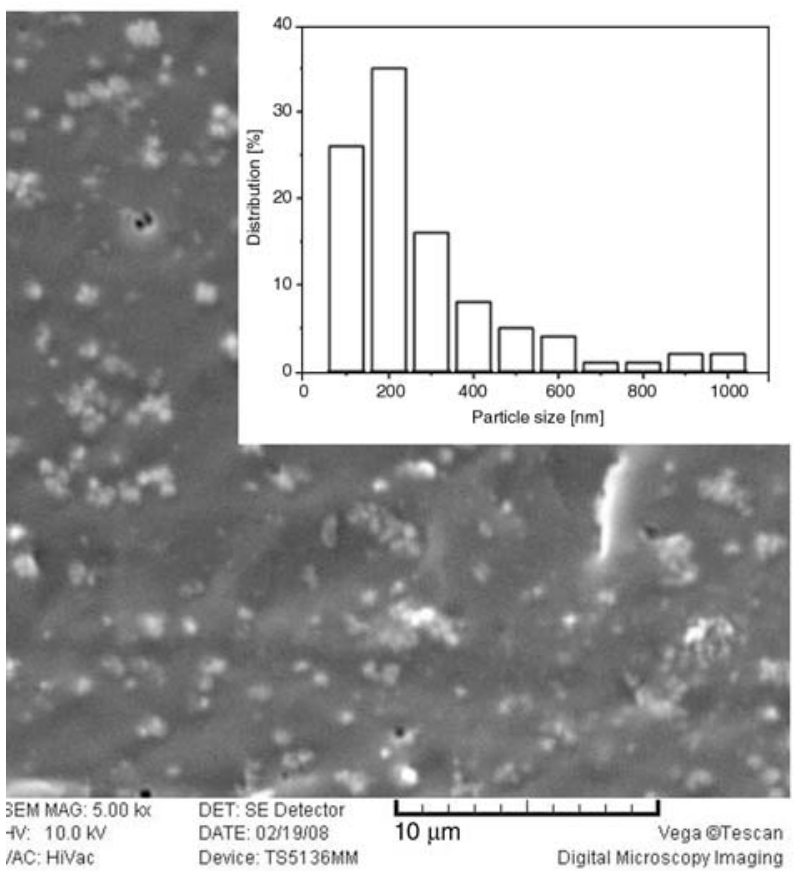

c) to modify the surface of anatase titania nanoparticles is feasible, and MAA can successfully improve the interfacial interactions between titania and PMMA matrix. Therefore, PMMA composite materials with enhanced thermal properties can be conveniently prepared by photocatalytic polymerization using the anatase titania sol.

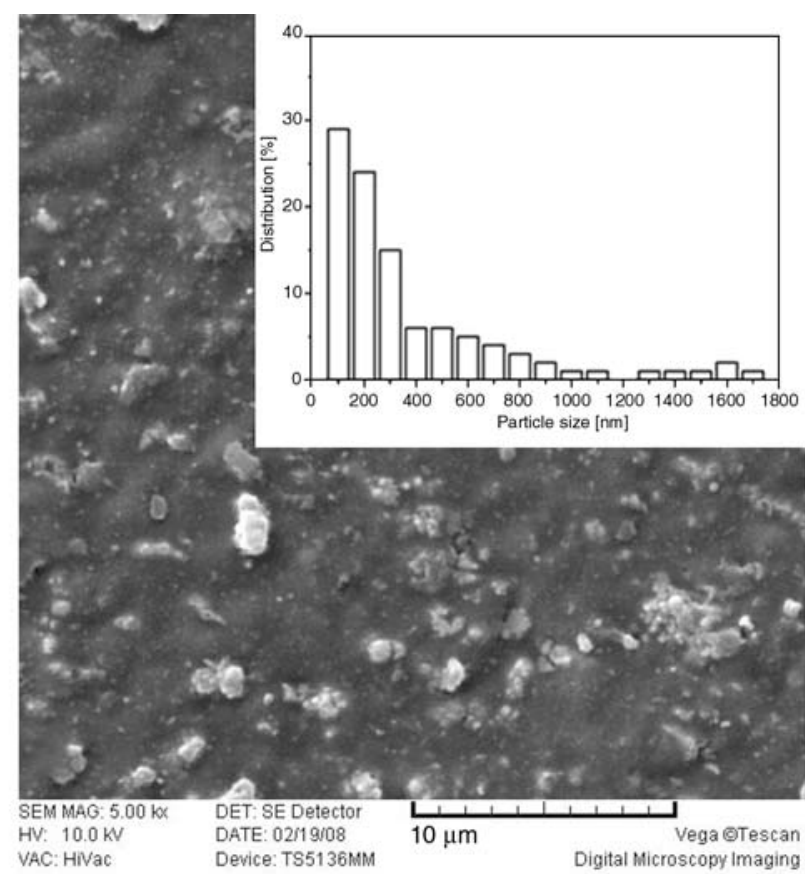

b)
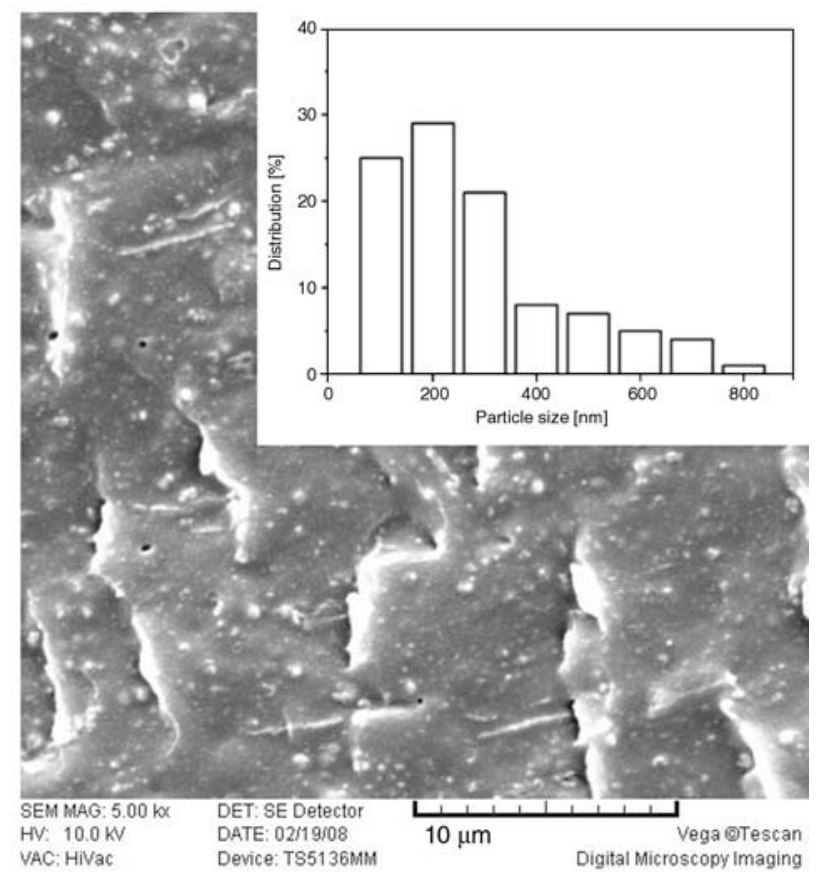

d)

Figure 7. SEM images of the PMMA/titania composite films (a) prepared via subsequent addition of titania and in situ polymerization with different MAA contents: (b) 0, (c) 10 and (d) $20 \mathrm{wt} \%$ 


\subsection{Morphology of PMMA/titania composite films}

SEM observations (Figure 7) were performed to examine the dispersion of the functionalized titania nanoparticles in the PMMA matrix. The fractured surface of the PMMA/titania composite film prepared via subsequent addition of the as-synthesized titania nanoparticles to PMMA solution was also characterized for comparison (Figure 7a). It is clearly found that the titania particles aggregate seriously and even deposit to the bottom of the PMMA composite film. The PMMA/titania composite films prepared via in situ polymerization have a much better distribution of inorganic particles as shown in Figure 7b-7d. For the better observation of dispersion of titania nanoparticles in PMMA matrix, the particle size and particle distribution of the PMMA/titania composites are also shown in Figure $7 b-7 d$. The PMMA composite with non-functionalized titania indicates the obvious aggregation of inorganic particles and their distribution in the PMMA matrix is relatively poor (Figure 7b). The size of about $67 \%$ titania particles is less than $300 \mathrm{~nm}$, while a portion of inorganic component is larger than $1 \mu \mathrm{m}$. To some extent, the aggregation of titania nanoparticles is restricted by the preparation method of in situ photocatalytic polymerization. The dispersion of the titania particles modified with 10 and $20 \mathrm{wt} \%$ MAA coupling agent (Figure 7c and 7d) is improved significantly in PMMA matrix. Above $75 \%$ titania particles are less than $300 \mathrm{~nm}$ in size and no inorganic particles larger than $1 \mu \mathrm{m}$ is found. The PMMA/titania composite with $20 \mathrm{wt} \%$ MAA shows a better distribution of titania particles in the PMMA matrix than the one with $10 \mathrm{wt} \%$ MAA. This may result from the existence of stronger interfacial interactions between polymer matrices and functionalized inorganic particles. However, these SEM observations of PMMA/titania composite films obviously deviate from our expectation because of the disagreement between the size of original titania nanoparticles $(\sim 20 \mathrm{~nm})$ and the inorganic nanoparticles in PMMA matrices (hundreds of nanometers) even after the surface modification. This may be clarified by the reaction process of the photocatalytic polymerization of the monomer. Our tentative pro- posed mechanism for the aggregation of anatase titania nanoparticles is as follows. The coupling agent MAA is initially absorbed onto the titania nanoparticles via the bidentate coordination. This leads to surface polymerization of MMA, which produces hydrophobic patches on the titania nanoparticles. In our reaction system, oil component (MMA) is excessive compared with its soluble amount in water and there must be the interfaces of oil/water during stirring. It has been proved that the energy of attachment of a spherical nanoparticle to the oil/water interface is related to the wettability of the nanoparticles and the nanoparticles with intermediate hydrophobicity benefit to stabilize oil drops in water [26]. Therefore, this leads to the movement of hydrophobic titania nanoparticles and their aggregation on the interfaces of oil/water to form the structure similar with Pickering emulsion.

\section{Conclusions}

A transparent anatase titania aqueous sol is successfully prepared by a facile nonhydrolytic sol-gel reaction. The PMMA/titania composites are prepared by the photocatalytic polymerization using functionalized anatase titania sol. According to the DSC, TGA and SEM results, introduction of MAA is beneficial to improve the interfacial interaction between PMMA matrices and titania nanoparticles. The glass transition temperatures and thermal stabilities of the PMMA/titania composites are increased. Compared with direct blending, the PMMA/titania composite films prepared via in situ polymerization show a much better distribution of titania particles. The partial aggregation of titania nanoparticles in PMMA composite films is caused by the enhancement of hydrophobicity of inorganic nanoparticles and formation of the structure like Pickering emulsion.

\section{Acknowledgements}

The authors gratefully acknowledge the financial support by the National Natural Science Foundation of China (NSFC) (No. 20704007) and Shanghai Nano Technology Project of China (No. 0625NM028). 


\section{References}

[1] Guidi V., Carotta M. C., Ferroni M., Martinelli G., Paglialonga L., Comini E., Sberveglieri G.: Preparation of nanosized titania thick and thin films as gassensors. Sensors and Actuators B: Chemical, 57, 197200 (1999).

DOI: $10.1016 / \mathrm{S} 0925-4005(99) 00080-5$

[2] Feldmann C.: Preparation of nanoscale pigment particles. Advanced Materials, 13, 1301-1303 (2001). DOI: $10.1002 / 1521-4095(200109) 13: 17<1301::$ AID ADMA1301>3.0.CO;2-6

[3] Kamat P. V., Dimitrijević N. M.: Colloidal semiconductors as photocatalysts for solar energy conversion. Solar Energy, 44, 83-98 (1990). DOI: $10.1016 / 0038-092 X(90) 90070-S$

[4] Grätzel M.: Photoelectrochemical cells. Nature, 414, 338-344 (2001). DOI: $10.1038 / 35104607$

[5] Legrini O., Oliveros E., Braun A. M.: Photochemical processes for water treatment. Chemical Reviews, 93, 671-698 (1993).

DOI: $10.1021 / \mathrm{cr} 00018 \mathrm{a} 003$

[6] Linsebigler A. L., Lu G. Q., Yates J. T.: Photocatalysis on $\mathrm{TiO}_{2}$ surfaces: Principles, mechanisms, and selected results. Chemical Reviews, 95, 735-758 (1995). DOI: $\underline{10.1021 / \mathrm{cr} 00035 \mathrm{a} 013}$

[7] Ni X. Y., Ye J., Dong C.: Kinetics studies of methyl methacrylate photopolymerization initiated by titanium dioxide semiconductor nanoparticles. Journal of Photochemistry and Photobiology A: Chemistry, 181, 19-27 (2006).

DOI: $10.1016 /$ j.jphotochem.2005.10.021

[8] Damm C., Völtzke D., Abicht H. P., Israel G.: Influence of the properties of $\mathrm{TiO}_{2}$ particles on a photocatalytic acrylate polymerization. Journal of Photochemistry and Photobiology A: Chemistry, 174, 171-179 (2005).

DOI: $\underline{10.1016 / \text { j.jphotochem.2005.03.006 }}$

[9] Damm C.: An acrylate polymerisation initiated by iron doped titanium dioxide. Journal of Photochemistry and Photobiology A: Chemistry, 181, 297-305 (2006). DOI: 10.1016/j.jphotochem.2005.12.011

[10] Yuwono A. H., Xue J. M., Wang J., Elim H. I., Ji W., Li Y., White T. J.: Transparent nanohybrids of nanocrystalline $\mathrm{TiO}_{2}$ in PMMA with unique nonlinear optical behavior. Journal of Materials Chemistry, 13, 1475-1479 (2003). DOI: 10.1039/b211976e

[11] Yang Y. N., Wang P.: Preparation and characterizations of a new $\mathrm{PS} / \mathrm{TiO}_{2}$ hybrid membranes by sol-gel process. Polymer, 47, 2683-2688 (2006).

DOI: $\underline{10.1016 / j . p o l y m e r .2006 .01 .019}$
[12] Goto K., Tamura J., Shinzato S., Fujibayashi S., Hashimoto M., Kawashita M., Kokubo T., Nakamura T.: Bioactive bone cements containing nano-sized titania particles for use as bone substitutes. Biomaterials, 26, 6496-6505 (2005).

DOI: $10.1016 /$ j.biomaterials.2005.04.044

[13] Ma D. L., Hugener T. A., Siegel R. W., Christerson A., Mårtensson E., Önneby C., Schadler L. S.: Influence of nanoparticle surface modification on the electrical behaviour of polyethylene nanocomposites. Nanotechnology, 16, 724-731 (2005).

DOI: $10.1088 / 0957-4484 / 16 / 6 / 016$

[14] Yoshida K., Taira Y., Atsuta M.: Properties of opaque resin composite containing coated and silanized titanium dioxide. Journal of Dental Research, 80, 864 868 (2001). DOI: $10.1177 / 00220345010800030401$

[15] Sidorenko A., Minko S., Gafijchuk G., Voronov S.: Radical polymerization initiated from a solid substrate. 3. grafting from the surface of an ultrafine powder. Macromolecules, 32, 4539-4543 (1999). DOI: $\underline{10.1021 / \mathrm{ma} 981355 \mathrm{u}}$

[16] Erdem B., Sudol E. D., Dimonie V. L., El-Aasser M. S.: Encapsulation of inorganic particles via miniemulsion polymerization. I. Dispersion of titanium dioxide particles in organic media using OLOA 370 as stabilizer. Journal of Polymer Science Part A: Polymer Chemistry, 38, 4419-4430 (2000).

DOI: 10.1002/1099-0518(20001215)38:24<4419:: AID-POLA110>3.0.CO;2-X

[17] Erdem B., Sudol E. D., Dimonie V. L., El-Aasser M. S.: Encapsulation of inorganic particles via miniemulsion polymerization. II. Preparation and characterization of styrene miniemulsion droplets containing $\mathrm{TiO}_{2}$ particles. Journal of Polymer Science Part A: Polymer Chemistry, 38, 4431-4440 (2000).

DOI: 10.1002/1099-0518(20001215)38:24<4431:: AID-POLA120>3.0.CO;2-Y

[18] Rong Y., Chen H-Z., Wu G., Wang M.: Preparation and characterization of titanium dioxide nanoparticle/polystyrene composites via radical polymerization. Materials Chemistry and Physics, 91, 370-374 (2005).

DOI: 10.1016/j.matchemphys.2004.11.042

[19] Khaled S. M., Sui R. H., Charpentier P. A., Rizkalla A. S.: Synthesis of $\mathrm{TiO}_{2}$-PMMA nanocomposite: Using methacrylic acid as a coupling agent. Langmuir, 23, 3988-3995 (2007). DOI: $10.1021 / \mathrm{la} 062879 \mathrm{n}$

[20] Yamazaki S., Nakamura N.: Photocatalytic reactivity of transparent titania sols prepared by peptization of titanium tetraisopropoxide. Journal of Photochemistry and Photobiology A: Chemistry, 193, 65-71 (2008). DOI: 10.1016/j.jphotochem.2007.06.008 
[21] Hojjati B., Sui R. H., Charpentier P. A.: Synthesis of $\mathrm{TiO}_{2} / \mathrm{PAA}$ nanocomposite by RAFT polymerization. Polymer, 48, 5850-5858 (2007).

DOI: 10.1016/j.polymer.2007.07.054

[22] Rotzinger F. P., Kesselman-Truttmann J. M., Hug S. J., Shklover V., Gratzel M.: Structure and vibrational spectrum of formate and acetate adsorbed from aqueous solution onto the $\mathrm{TiO}_{2}$ rutile (110) surface. Journal of Physical Chemistry B, 108, 5004-5017 (2004). DOI: 10.1021/jp0360974

[23] Manring L. E.: Thermal degradation of saturated poly(methyl methacrylate). Macromolecules, 21, 528-530 (1988).

DOI: $\underline{10.1021 / \mathrm{ma} 00180 \mathrm{a} 046}$
[24] Chang T. C., Wang Y. T., Hong Y. S., Chiu Y. S.: Organic-inorganic hybrid materials. V. Dynamics and degradation of poly(methyl methacrylate) silica hybrids. Journal of Polymer Science Part A: Polymer Chemistry, 38, 1972-1980 (2000).

DOI: $10.1002 /($ SICI $) 1099-0518(20000601) 38: 11$ $\leq 1972:$ :AID-POLA60>3.0.CO;2-5

[25] Wang H. T., Meng S., Xu P., Zhong W., Du Q. G.: Effect of traces of inorganic content on thermal stability of poly(methyl methacrylate) nanocomposites. Polymer Engineering and Science, 47, 302-307 (2007). DOI: $10.1002 /$ pen. 20708

[26] Binks B. P., Lumsdon S. O.: Influence of particle wettability on the type and stability of surfactant-free emulsions. Langmuir, 16, 8622-8631 (2000). DOI: $\underline{10.1021 / \mathrm{la} 000189 \mathrm{~s}}$ 